\title{
Nachruf auf David A. Kipper
}

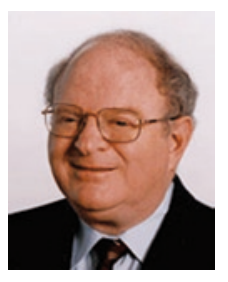

Nach schwerer Krankheit ist der weltweit bedeutende Psychodramatiker David A. Kipper im Alter von 71 Jahren am 2. Dezember 2010 in Chicago im Kreis seiner Familie verstorben.

Geboren wurde David 1939 in Tel Aviv. Er studierte Psychologie und erwarb 1964 den B.A. an der Bar Ilan Universität, Israel und 1969 an der University of Durham, England den $\mathrm{PhD}$. Eher zufällig stieß er auf das Psychodrama und lernte 1966 J.L. und Zerka bei der Internationalen Psychodramatagung in Barcelona kennen. Anschließend bis 1967 organisierte David einen Aufenthalt als Ausbildungskandidat an der Moreno Akademie für Gruppenpsychotherapie und Psychodrama in Beacon, New York.

Seine akademische Karriere begann in Israel, wo er in verschiedenen Rollen an seiner Stammuniversität tätig war. Schon 1972 bis 1973 setzte er einen Fuß in die staatliche Universität von New York und ab 1984 war er an der Universität von Chicago tätig. Die letzten fünfzehn Jahre war er Forschungsprofessor an der Roosevelt Universität in Chicago und leitete das Institut für Gruppenforschung und Ausbildung. Die universitäre Lehre führte ihn auch an die Loyola Universität in Chicago, die Universität von Tel Aviv und die Freie Universität von Moskau. Vorträge hielt er darüber hinaus in Kanada, Mexiko, Brasilien, Argentinien, in der Türkei, in Bulgarien, Rumänien, Taiwan und Japan. In Israel baute er ein Musiktherapieprogramm mit auf.

Neben der klinischen Psychologie und Psychotherapie hatte David Kipper eine starke Fundierung in der Managementberatung, die er innerhalb der Universität und in einer eigens gegründeten und von ihm geleiteten Firma (http://www.execpsych.com/) einbrachte. Er erhielt zahlreiche Zertifikate als Psychologe, Supervisor, Gruppenpsychotherapeut und Psychodrama-Ausbilder in den USA und Israel.

Mehr als dreißig Jahre war es David ein Anliegen, gute wissenschaftliche Publikationen in Israel und USA zu ermöglichen. Dazu war er in zahlreichen Zeitschriften des angesehenen Sage Verlags und der Amerikanischen Psychologischen Vereinigung (APA)

Online publiziert: 02.02 .2011

(C) VS Verlag für Sozialwissenschaften 2011 
als Redaktionsmitglied tätig. Das von J.L. Moreno begründete „Journal of Group Psychotherapy, Psychodrama, and Sociometry“ gab er fünfzehn Jahre mit heraus und war darüber hinaus sieben Jahre beratend tätig.

Herauszuheben ist Davids Engagement in professionellen Vereinigungen. Unter anderem stand er der Israelischen Psychologischen Vereinigung (IPA), der schon genannten APA im Bereich der Gruppenpsychologie und Psychotherapie, der Amerikanischen Akademie für Gruppenpsychologie (AAGP), der Amerikanischen Gesellschaft für Gruppenpsychotherapie und Psychodrama (ASGPP) vor und gründete mit anderen Beteiligten die Psychodrama-Sektion und ein Jahrbuch in der Internationalen Vereinigung für Gruppenpsychotherapie und Gruppenprozesse (IAGP).

Seine Veröffentlichungen sind schier unerschöpflich. Schon 1967 publizierte er zum Thema Spontaneität, was ab 2005 zum „,The Spontaneity Assessment Inventory (SAI-R)“ führte, an dem er bis zum Tod gearbeitet hat. Wir werden in einem Sonderheft sein letztes Manuskript vom November 2010 übersetzen. 1968 gab es einen gemeinsamen Buchbeitrag mit J.L. Moreno und 1990 gemeinsam mit Zerka. Das Buch „Psychotherapy Through Clinical Role Play“ (1986) wurde in russisch (1993) und bulgarisch (2001) übersetzt. Neben den genannten Sprachen gibt es noch Übersetzungen von Artikeln in die italienische und türkische Sprache, möglicherweise noch andere, da er nicht alle dokumentierte (s. http://www.davidakipper.com/publications/).

1989 und 2003 überprüfte er die Effektivität des Psychodramas, letzteres wurde in dieser Zeitschrift 2004 ins Deutsche übertragen, und gab im selben Jahr eine Übersicht von klinischen Fallstudien. Im letzten Jahrzehnt hat David Kipper das „Experiential Action Reintegration Model (EARM)“ entwickelt. Er vertrat den Standpunkt, dass das Psychodrama auch isoliert als Methode ohne die dahinterliegende Theorie verwendet werden kann.

Davids Interessenkreis ging aber weit über den Wissenschaftsbetrieb hinaus. Er übernahm führende Funktionen im Kulturbereich von Chicago wie im Komödientheater, beim Joffrey Ballett, im College für Darstellende Kunst und für das Symphonie Orchester. Herausragend war auch sein Einsatz für jüdische Organisationen wie u. a. den „American Jewish Congress“.

Für sein Lebenswerk erhielt er zahlreiche Auszeichnungen vom „American Board of Professional Psychology“ (ABPP), der „Anti-Defamation League (ADL)“, der „National Foundation of Jewish Culture“ und von der genannten ASGPP. In letzterer wurde seit 1994 ein „Award“ nach ihm benannt.

David hat seine Krebserkrankung - so gut es ging - unaufgeregt behandelt. Er hinterlässt seine Frau Barbara Levy Kipper (http://blkipper.com/), die er in Beacon kennengelernt hatte, die Töchter Talia und Tamar und die Enkel Anya Rose und Eli. Ein Kondolenzbuch ist im Internet eingerichtet unter http:/www.legacy.com/guestbook/chicagotribune/guestbook.aspx?n=david-kipper\&pid=146911430\&view=2\&entry=461601 97. Das Mitgefühl gilt den Hinterbliebenen.

Seine Person kann man als intelligent, weise, engagiert, fair, Wärme ausstrahlend und großzügig beschreiben. Das konnte ich selbst bei Begegnungen anlässlich von Tagungen und anhand unseres E-Mail-Verkehrs erfahren. Es gibt wenige Universitätsprofessoren, die sich auf das Psychodrama konzentrieren, deshalb ist sein Verlust für unsere Fachwelt besonders groß. David war eine Leitfigur, ein Vorbild und eine Brücke zur akademischen 
Welt. Wir hatten in den letzen fünf Semestern Lehrveranstaltungen und einen Gastvortrag in Klagenfurt für ihn geplant, aber er musste sie krankheitshalber verschieben - es sollte nicht mehr dazu kommen. Die Lehrveranstaltungsbeschreibung ist uns als Andenken geblieben.

Auch in der FEPTO (Federation of European Psychodrama Training Organizations) hatten wir im Forschungskomitee noch viel mit ihm vor. David ist während Hanukkah verstorben, sein Licht kann uns den Weg weisen. In einem Interview mit M. Malenov aus dem Jahre 2003 sagte er in seiner humorvollen Art über das Psychodrama: „I still think that the most important is how to handle the chairs! (Laughter). The rest will be ...". Und Zerka fügt nun an: „There will always be an empty chair for David.“

Michael Wieser, Klagenfurt 